\title{
Optimizing Instantaneous and Ramping Reserves with Different Response Speeds for Contingencies-Part II: Implications
}

\author{
Josh Schipper, Alan Wood, Member, IEEE, and Conrad Edwards
}

\begin{abstract}
Part I presents a formulation to optimize reserves for contingent events while explicitly including their response times. Part II highlights the implications of using this formulation in a reserves market. There are four aspects of the formulation that are of interest: (1) the performance of the solver, (2) the importance of inertia and contingency size. These implications are highlighted through two examples. (3) The proposed formulation is compared against a reserve optimization which does not distinguish between different response speeds, but adjusts the reserve requirement to satisfy the same frequency limits. Differentiating between response speeds within the optimization can provide a $23 \%$ reduction in total reserve requirement on average, which improves to a reduction of $52 \%$ in low inertia conditions. (4) A marginal pricing methodology is developed which prices reserve in a market context, and gives a unique price to each reserve provider depending on response speed. This gives incentive for reserve providers to improve response speed.
\end{abstract}

Index Terms-Reserve Markets, Primary Frequency Control Reserve, Contingency Reserve, Quadratically Constrained Programming, Convex Optimization.

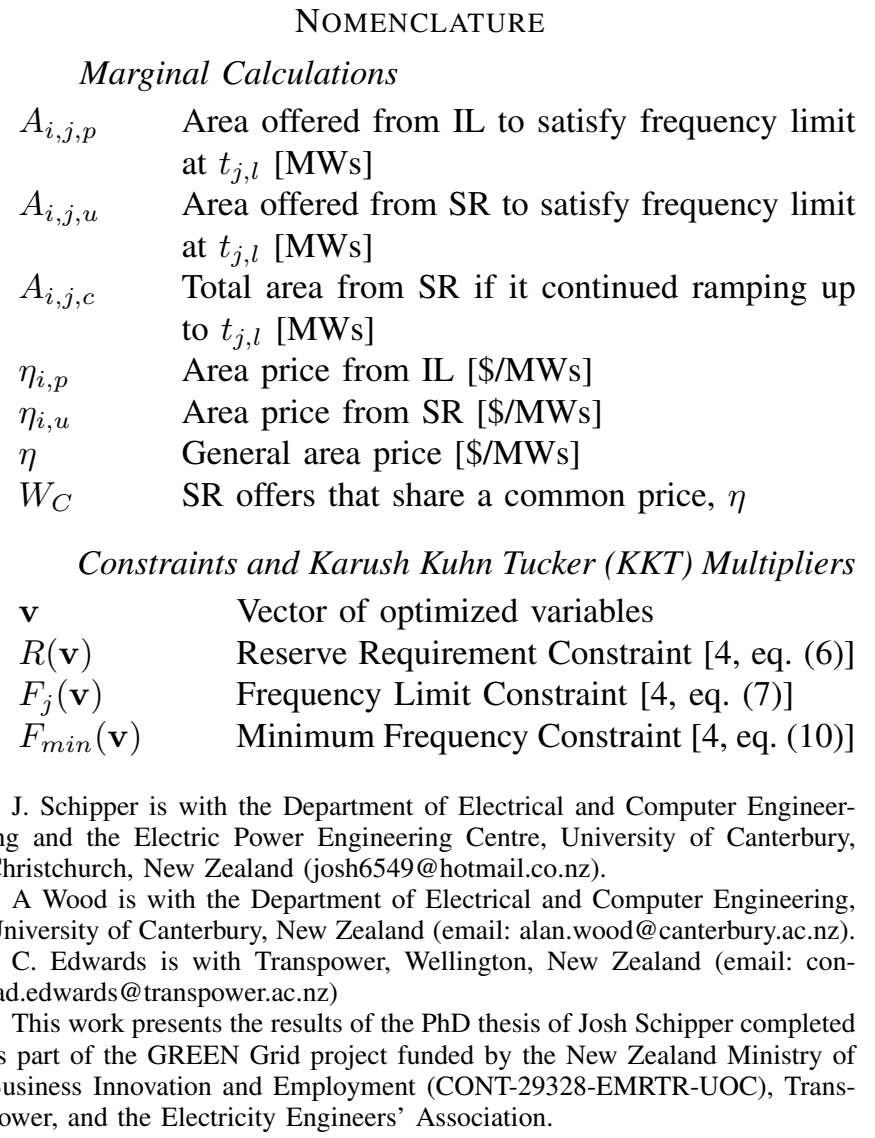

$\begin{array}{ll}\mathbf{G}\left(f_{k, \lambda}, t_{k}, \boldsymbol{\tau}\right) & \text { Gradient vector for frequency constraints } \\ \nu & \text { KKT multiplier for } R(\mathbf{v}) \leq 0[\$ / \mathrm{MW}] \\ \lambda_{j, l} & \text { KKT multiplier for } F_{j}(\mathbf{v}) \leq 0[\$ / \mathrm{MWs}] \\ \lambda_{\min } & \text { KKT multiplier for } F_{\min }(\mathbf{v}) \leq 0[\$ / \mathrm{MW}] \\ \lambda_{\min , j} & \text { other KKT multipliers for } F_{\min }(\mathbf{v}) \leq 0 \\ & \text { when nonsmooth and continuous [\$/MWs] } \\ \lambda_{k} & \text { Generalized KKT multipliers for frequency } \\ & \text { constraints [\$/MWs] } \\ \boldsymbol{\mu}^{\min } & \text { KKT multipliers for minimum limits } \\ \boldsymbol{\mu}^{\max } & \text { KKT multipliers for maximum limits }\end{array}$

$\quad$ Time,
$t_{\text {min }}$
$t_{\text {min,j }}$
$\Omega$
$\tau, \tau_{i}, \boldsymbol{\tau}$
$t_{k}$
$f_{\text {lim,j }}$
$f_{k, \lambda}$
$c_{i}$
$\mathbf{c}$
$c(\tau)$
$c_{R}$
$c_{H}$

Time, Frequency, and Prices

Time of minimum frequency [s]

Times the minimum frequency can jump to from point $\mathbf{v}[\mathrm{s}]$

Set of possible $t_{\text {min,j }}$

$\tau, \tau_{i}, \tau \quad$ Time(s) a unit of reserve is offered [s]

$t_{k} \quad$ Time where frequency constraint is evaluated [s]

$f_{\text {lim }, j} \quad$ Equivalent to $f_{\text {lim }}\left(t_{\text {min }, j}\right)$

$f_{k, \lambda} \quad$ Equivalent to $f_{\text {lim }}\left(t_{k}\right)$

$c_{i} \quad$ General price for offered reserve [\$/MW]

Price vector corresponding to $\mathbf{v}$

$(\tau) \quad$ Marginal reserve price function [\$/MW]

Marginal cost of increasing $R[\$ / \mathrm{MW}]$
Marginal value of increasing $H$ [\$/MWs]

\section{INTRODUCTION}

$\mathbf{R}$ ESERVE Markets efficiently procure reserve to satisfy the security requirements of the power system, and ultimately to facilitate the transfer of electricity. Therefore it is important that reserve markets are properly designed so that electricity transfer continues without repeated interruptions and with minimal cost, so that the consumer has a low cost and reliable energy source. However, Variable Renewable Energy (VRE) is changing the requirements for reserves, particularly in small power systems that have low inertia and are more susceptible to power imbalances causing fluctuations in grid frequency. VRE decreases the total inertia on these power systems, creating a requirement for faster reserves. The power systems of Ireland [1], Great Britain [2], and Eastern Australia [3] are introducing new reserve categories for the procurement of faster reserves. An optimization formulation is developed in the companion paper [4], that removes the definitions of specific reserve categories and replaces them with models of instantaneous and ramped responses, so that choices can be made between reserves with different speeds and different 
prices. This paper discusses the implications of using this optimization formulation on reserve market design by way of examples.

Reserve markets, as one means of Ancillary Services procurement, require careful design. Following [5], some of these design aspects include the auction structure, offer forming, optimization formulation and methodology, the sequencing of auction events and information dissemination, pricing methodology and clearing mechanism; market duration, cost allocation, and the settlement process. To simplify the discussion, only new aspects of the optimization formulation are discussed.

There are four aspects of the methodology that are analyzed in detail. (1) Optimizations for real-time electricity markets need to be executable within the dispatch interval, e.g. every five minutes in New Zealand [6]. Since this new formulation does not use MILP, it needs to be demonstrated that the solve time remains practical. (2) The methodology can include the inertial constant and the largest credible contingency, i.e. risk, as variables in the optimization, and it is discussed when these options should be applied. (3) A comparison is made with an optimization formulation that only considers reserve capacity within the optimization, but adjusts the reserve requirement above the size of the contingency to satisfy the same frequency constraints. This shows the improvement that considering response times has in the optimization. (4) A pricing methodology is developed, where the reserve price is a function of time when a unit of reserve responds. This is to value different response speeds separately, providing incentive for reserve providers to improve their response characteristics. Before the pricing methodology is developed, to improve the transparency of how the global minimum and prices are found, it is shown how a merit-order curve is formed when one frequency limit is binding.

This paper is organized as follows. Section III solves an example with frequency limits to highlight their importance. Section III presents a simplified method of predicting the optimal solution and uses the first example as a test case. Section IV shows the performance of the solver for the first example. Section $\mathrm{V}$ presents a second example for which inertia and risk are varied, so that the cost in changes to these variables is analyzed. Section VI compares a simplified way of optimizing reserve. Section VII develops the pricing methodology. Lastly, Section VIII briefly discusses reserve response modeling.

\section{EXAMPLE 1 - FREQUENCY Limits}

This section shows how a problem is solved with multiple frequency limits. A problem is created with multiple IL and SR offers, as listed in Table I] These offers do not represent any particular power system. The inertial constant is 15,000 MWs and the risk is $400 \mathrm{MW}$, these values are typical for the North Island of New Zealand. An initial frequency limit of 48 $\mathrm{Hz}$ is included with frequency limits of Table III. The steps in finding the global minimum are shown in Table III and the frequency transients in Fig. 1 .

The identification of regions is specified by a triple, as labeled in Table IIII and Fig. 1. The first number specifies
TABLE I

LIST OF IL AND SR OFFERS FOR THE FIRST EXAMPLE

\begin{tabular}{c|cccc|} 
& Quantity (MW) & Price (\$/MW) & $t_{i, p} / t_{i, u}(\mathrm{~s})$ & $g_{i}(\mathrm{MW} / \mathrm{s})$ \\
\hline & \multicolumn{5}{|c|}{ IL Offers } \\
1 & 10 & 150 & 0.9 & - \\
2 & 38 & 126 & 0.9 & - \\
3 & 16 & 0 & 1 & - \\
4 & 57 & 65 & 1 & - \\
5 & 42 & 132 & 1.1 & - \\
6 & 63 & 118 & 1.3 & - \\
7 & 29 & 98 & 1.5 & - \\
8 & 50 & 100 & 2.5 & - \\
9 & 75 & 50 & 3 & - \\
10 & 18 & 20 & 3.3 & - \\
\hline & \multicolumn{5}{|c}{ SR Offers } & 1.2 & \\
1 & 90 & 80 & 1.3 & 8 \\
2 & 32 & 160 & 1.3 & 2 \\
3 & 10 & 100 & 1.4 & 25 \\
4 & 200 & 5 & 1.4 & 15 \\
5 & 62 & 0 & 1.5 & 5 \\
6 & 25 & 10 & 1.5 & 6 \\
7 & 56 & 40 & 1.6 & 12 \\
8 & 81 & 50 & 1.7 & 1 \\
9 & 8 & 84 & 2 & 6 \\
10 & 27 & 18 & &
\end{tabular}

which range $t_{\min }$ is in (the smaller the number, the earlier $\left.t_{\text {min }}\right)$, the second number specifies the partitioning of $W_{B}$ and $W_{T}$, and the third specifies the partitioning of $W_{B, j}$ and $W_{T, j}$. The progress of the solver is seen by the first number increasing, as less expensive dispatches are found with later times of minimum frequency. This is recognized by the reducing total cost shown at the bottom of Table III

TABLE II

THE FREQUENCY LIMITS ADDED TO THE FIRST EXAMPLE.

\begin{tabular}{c|c}
\hline Time, $t_{j, l},($ seconds) & Frequency $(\mathrm{Hz})$ \\
\hline 8 & 48.75 \\
9 & 49.35 \\
10.5 & 49.6 \\
12 & 49.8
\end{tabular}

The frequency limits, as seen in Fig. 1. are important for the frequency to return to $50 \mathrm{~Hz}$, and is done in slightly over 11 seconds. The total reserve dispatched is $581.90 \mathrm{MW}$, i.e. dispatching extra reserve to achieve a positive derivative in the frequency transient.

The validity of the dispatch solution to model the frequency transient is limited to the first swing in that transient. Any time after this, the frequency limits and reserve offers are not appropriate to model subsequent control actions.

It should be mentioned that the frequency transient is based on the Center of Inertia (COI) frequency for a power system, and the localized frequency transient may experience oscillatory deviations from the COI frequency. Therefore it is possible that limiting the COI frequency in the optimization may not ensure the bounding of frequency in all locations. The size and period of these oscillations is dependent on each individual power system. For small power systems like New Zealand, these oscillations do not dominate the overall trend in the COI frequency. For potential oscillations that do occur, a factor of safety can be added to the frequency limits. For larger power systems, the proposed reserve optimization's 
TABLE III

RESULTS FOR FIRST EXAMPLE WITH ADDITIONAL FREQUENCY CONSTRAINTS

\begin{tabular}{|c|c|c|c|c|c|}
\hline $\begin{array}{c}\text { Step } \\
\text { Triple }\end{array}$ & $\begin{array}{c}1 \\
(1,1024,72)\end{array}$ & $\begin{array}{c}2 \\
(2,472,32) \\
\end{array}$ & $\begin{array}{c}3 \\
(4,893,4)\end{array}$ & $\begin{array}{c}4 \\
(6,216,1)\end{array}$ & $\begin{array}{c}5 \\
(6,760,2)\end{array}$ \\
\hline & \multicolumn{5}{|c|}{ IL Dispatch (MW) } \\
\hline 1 & 3.9 & 0 & 0 & 0 & 0 \\
\hline 2 & 38 & 38 & 0 & 0 & 0 \\
\hline 3 & 16 & 16 & 16 & 16 & 16 \\
\hline 4 & 57 & 57 & 57 & 57 & 57 \\
\hline 5 & 42 & 12 & 0 & 0 & 0 \\
\hline 6 & 63 & 63 & 11.9 & 0 & 0 \\
\hline 7 & 29 & 29 & 29 & 29 & 25.83 \\
\hline 8 & 50 & 50 & 50 & 0 & 0 \\
\hline 9 & 0 & 0 & 75 & 75 & 75 \\
\hline \multirow[t]{2}{*}{10} & 0 & 0 & 0 & 18 & 18 \\
\hline & \multicolumn{5}{|c|}{ SR Dispatch (MW) } \\
\hline 1 & 19.5 & 27 & 31.5 & 31.91 & 25.16 \\
\hline 2 & 9.6 & 0 & 0 & 0 & 0 \\
\hline 3 & 2.4 & 3.4 & 4 & 1.55 & 0.09 \\
\hline 4 & 190 & 165 & 94.78 & 165 & 180.43 \\
\hline 5 & 39.25 & 43 & 62 & 62 & 62 \\
\hline 6 & 5 & 7.5 & 9 & 12.35 & 25 \\
\hline 7 & 54 & 45 & 39 & 29.96 & 26.63 \\
\hline 8 & 76.8 & 16.8 & 20.4 & 51.19 & 42.88 \\
\hline 9 & 7.3 & 6.3 & 1.6 & 1.38 & 0.87 \\
\hline \multirow[t]{5}{*}{10} & 3 & 6 & 7.8 & 11.81 & 27 \\
\hline & \multicolumn{5}{|c|}{ Total Dispatched Reserve (MW) } \\
\hline & 705.75 & 585 & 508.98 & 562.15 & 581.90 \\
\hline & \multicolumn{5}{|c|}{ Total Cost (\$) } \\
\hline & 40,901 & 32,030 & 23,040 & 18,399 & 17,289 \\
\hline
\end{tabular}

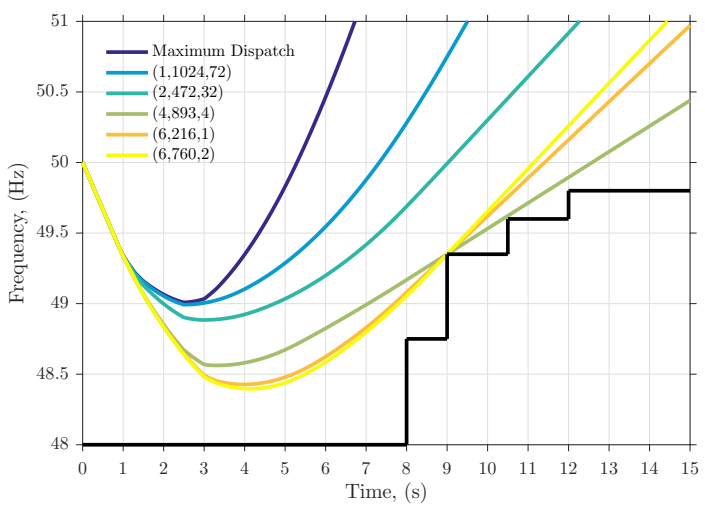

Fig. 1. The frequency transient for each local minimum in the steps to find the global minimum with frequency limits.

focus shifts from ensuring the frequency limit is satisfied in all locations, to ensuring that the rotational energy of all synchronous machines is restored on average.

\section{OfFer Curves ANd Marginal Providers}

The reserve dispatch of Example 1 does not follow the expected dispatch if only a reserve requirement is needed to be met. Otherwise SR offer 4 would be fully dispatched to $200 \mathrm{MW}$ as it is one of cheapest reserve. It is necessary to understand why the optimal solution results in the dispatch it creates, to help trust the solving methodology. It is difficult to analyze every situation, but it is possible to consider what is happening when there is only one binding frequency limit.

In Example 1, the optimal dispatch and its frequency transient result in the frequency limit at 9 seconds being the only binding constraint. In this instance it is possible to simplify the solving methodology by only considering this single frequency limit and the capacity constraints.

It is known that no reserve dispatched after the time of the frequency limit will contribute to satisfying the constraint. Therefore the frequency limits in the companion paper [4, eq. (21)], are simplified to:

$$
\begin{gathered}
\sum_{i \in Q_{B, j}} p_{i}\left(t_{j, l}-t_{i, p}\right)+\sum_{i \in W_{B, j}}\left(u_{i}\left(t_{j, l}-t_{i, u}\right)-\frac{u_{i}^{2}}{2 g_{i}}\right) \\
\geq R t_{j, l}+2 H f_{j}
\end{gathered}
$$

where $W_{B, j}$ is partitioned so that $W_{T, j}$ is empty, hence $W_{B, j}$ contains all possible elements. The optimization problem is to minimize the cost of reserve energy in MWs, instead of reserve capacity in MW. The energy reserve provides can be considered as the area below the reserve response curves, $P_{i}\left(t, p_{i}\right)$ and $U_{i}\left(t, u_{i}\right)$, therefore the terms 'energy' and 'area' are used synonymously. It will be shown that instead of forming a merit order curve with reserve quantity, one can be formed with reserve area. Firstly, the area terms are defined:

$$
\begin{gathered}
A_{i, j, p}\left(p_{i}\right)=p_{i}\left(t_{j, l}-t_{i, p}\right) \\
A_{i, j, u}\left(u_{i}\right)=u_{i}\left(t_{j, l}-t_{i, u}\right)-\frac{u_{i}^{2}}{2 g_{i}}
\end{gathered}
$$

To determine where each unit of area lies in the merit order, it is necessary to know the price for each unit. For a SR offer, the price of the next unit of area after $u_{i}$ of reserve is dispatched already is found by the following limit:

$$
\begin{gathered}
\eta_{i, u}\left(u_{i}\right)=\lim _{\Delta u_{i} \rightarrow 0} \frac{c_{i, u}\left(u_{i}+\Delta u_{i}\right)-c_{i, u} u_{i}}{A_{i, j, u}\left(u_{i}+\Delta u_{i}\right)-A_{i, j, u}\left(u_{i}\right)} \\
=\frac{c_{i, u}}{t_{j, l}-t_{i, u}-u_{i} / g_{i}}
\end{gathered}
$$

(5) is simplified by substituting in $u_{i}=g_{i}\left(\tau_{i}-t_{i, u}\right)$, where $\tau_{i}$ is the time that unit of area is offered:

$$
\eta_{i, u}\left(\tau_{i}\right)=\frac{c_{i, u}}{t_{j, l}-\tau_{i}}
$$

Following a similar process, the price for a unit of area from an IL offer is:

$$
\eta_{i, p}=\frac{c_{i, p}}{t_{j, l}-t_{i, p}}
$$

which is constant and independent of $p_{i}$. Next in creating the merit order curve is to formulate $\eta_{i, u}$ as a function of $A_{i, j, u}$. Therefore solving (3) for $u_{i}$ and substituting into (5):

$$
\eta_{i, u}\left(A_{i, j, u}\right)=c_{i, u} \sqrt{\frac{g_{i} / 2}{A_{i, j, c}-A_{i, j, u}}}
$$

where

$$
A_{i, j, c}=\frac{1}{2} g_{i}\left(t_{j, l}-t_{i, u}\right)^{2}
$$




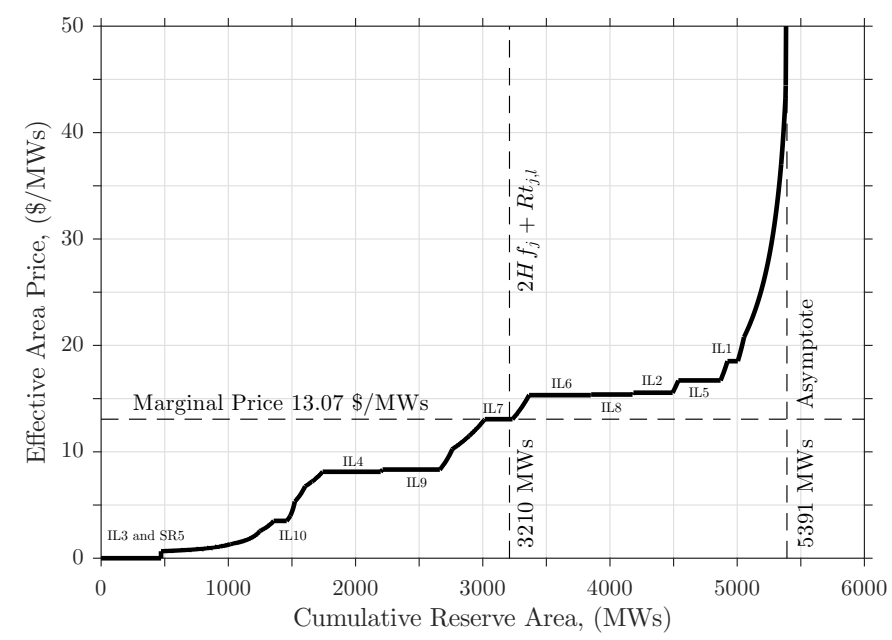

Fig. 2. The offer curve for reserve to satisfy the frequency limit at 9 seconds $(49.35 \mathrm{~Hz})$ in the first example.

Organizing area from least to most expensive the meritorder curve is produced. Fig. 2 shows merit-order curve for the offers of Example 1. The flat portions of the curve are from IL offers. Areas from SR offers are separated either side of IL offers, and are combined with other SR offers in these gaps, as SR offers can share the same price. The equation that describes the shape of the curve when multiple SR offers are combined is:

$$
\eta(\Delta A)=\sqrt{\frac{\frac{1}{2} \sum_{i \in W_{C}} g_{i} c_{i, u}^{2}}{\left(\sum_{i \in W_{C}} A_{i, j, c}-A_{i, j, u}\left(\eta_{0}\right)\right)-\Delta A}}
$$

where $W_{C}$ is the set of all SR offers that share the same price range for $\eta \geq \eta_{0}$ until one provider can no longer offer any more area.

For Fig. 2, the area requirement is $3210 \mathrm{MWs}$, this results in IL7 being the marginal offer with a price of $\$ 13.07 / \mathrm{MWs}$. Since SR offers 1, 3, 4, 7, 8, and 9 has \$13.07/MWs within their area price range these offers are partially dispatched, as only area below this price is required. This is seen in the partial dispatch of SR offers in Table III

\section{PERFORMANCE}

Computational performance is measured by how many regions are optimized. If these constraints were included with the energy market formulation, then solving each region would be comparable to solving the energy market once. Therefore in the time between dispatches, 5 minutes in NZ, it is important to keep the number of regions to solve as small as possible. This section compares how the estimates from the companion paper [4] with the actual number of regions solved.

The companion paper made two estimates: one for the total number of possible regions $N_{r}$, and the number of regions solved $N_{s}$. These are worst case estimates, assuming worst possible configuration of offers and timing of frequency limits. Therefore it should be expected that the actual numbers would be much less than this. For Example 1, the numbers are shown in Table IV, which show the actual numbers are significantly less than the estimates. Since the number of solved regions is below 10, it is expected that the impact on solve time for a co-optimized problem will be by one order of magnitude.

TABLE IV

PERFORMANCE OF SOLVING EXAMPLE 1

\begin{tabular}{c|c}
$N_{r}$ & $1,572,120,576$ \\
No. of Feasible Regions & 91,713 \\
$N_{s}$ & 43 \\
No. of Solved Regions & 5
\end{tabular}

Running this optimization on a Intel i7-8700 CPU, 3.20 $\mathrm{GHz}$, the solve time is 3.1 seconds. The solving methodology is scripted in MATLAB and uses a non-commercial QCP solver which is also scripted in MATLAB.

\section{EXAMPLE 2 - INERTIA AND RISK VARIATION}

[7] has demonstrated that inertial constant, $H$, and risk, $R$, can be variables in the optimization, as the feasible solution space will remain convex. However the solving methodology requires further development to incorporate inertia and risk with MILP formulations of the energy market. This section completes multiple optimizations with inertia and risk as constants, but varied for each optimization. The purpose of this is to show how optimal solutions change, and to show why and when it is appropriate to include inertia and risk as variables in the energy and reserve co-optimization.

A single set of offers is created for this example in Table V. A similar set of frequency limits is used from Example 1, Table II. except the time of each limit is delayed one second. There is a base inertia of 15,000 MWs and base risk of 400 MW unless they are varied. The reserve offers do not reflect any particular power system. Offer prices are correlated so that the earliest initiated reserve is also the most expensive.

TABLE V

LIST OF IL AND SR OFFERS FOR THE SECOND EXAMPLE

\begin{tabular}{c|cccc|} 
& Quantity (MW) & Price (\$/MW) & $t_{i, p} / t_{i, u}(\mathrm{~s})$ & $g_{i}(\mathrm{MW} / \mathrm{s})$ \\
\hline & \multicolumn{5}{|c|}{ IL Offers } \\
1 & 68 & 400 & 0.9 & - \\
2 & 16 & 300 & 1 & - \\
3 & 54 & 200 & 1 & - \\
4 & 152 & 160 & 1.2 & - \\
5 & 23 & 120 & 1.8 & - \\
6 & 89 & 80 & 2.5 & - \\
7 & 48 & 0 & 3.5 & - \\
\hline & 5 & SR Offers & \\
1 & 71 & 150 & 0.6 & 16 \\
2 & 26 & 130 & 0.8 & 4 \\
3 & 67 & 110 & 1.1 & 5 \\
4 & 62 & 90 & 1.2 & 12 \\
5 & 165 & 70 & 1.6 & 18 \\
6 & 47 & 50 & 2 & 6 \\
7 & 33 & 30 & 2.2 & 3 \\
8 & 28 & 10 & 3 & 6 \\
\hline
\end{tabular}

Inertia is varied from 6,500 MWs to 65,000 MWs. The lower value is slightly greater than the minimum possible value for this set of offers of 6,433 MWs. For selected inertia values within the range, the optimal solutions are shown in Table VI, and the resultant frequency transients in Fig. 3. The total 
cost and total dispatched reserve of the optimal solution as a function of inertia is shown in Fig. 4, labeled 'Optimal'.

The lowest possible value for risk is $0 \mathrm{MW}$, but for a practical outcome the range starts at $200 \mathrm{MW}$. The upper range finishes at $600 \mathrm{MW}$, below the maximum possible of 627 MW. The optimal solutions are presented in Table VII and the frequency transients in Fig. 5. The total cost and total dispatched reserve for variations in risk are shown in Fig. 6. labeled 'Optimal'.

The mean time to solve each optimization is $4.0 \mathrm{~s}$ for each variation in inertia and risk. The fastest solve is in $2.7 \mathrm{~s}$, while the slowest solve is in $6.6 \mathrm{~s}$.

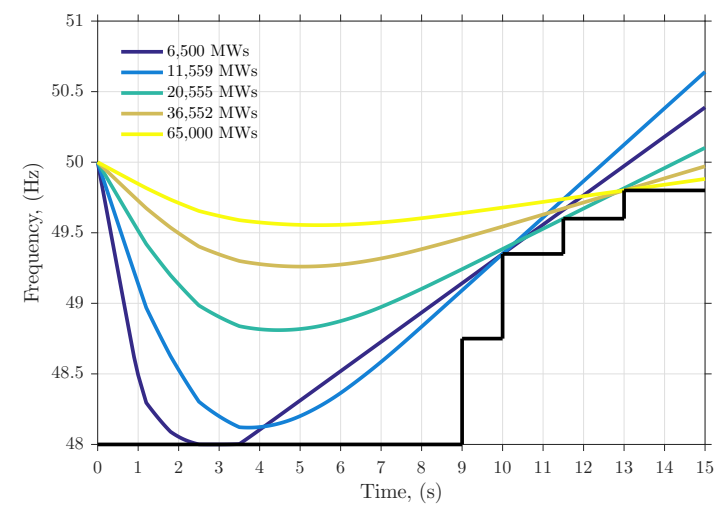

Fig. 3. Frequency transients for different inertia.

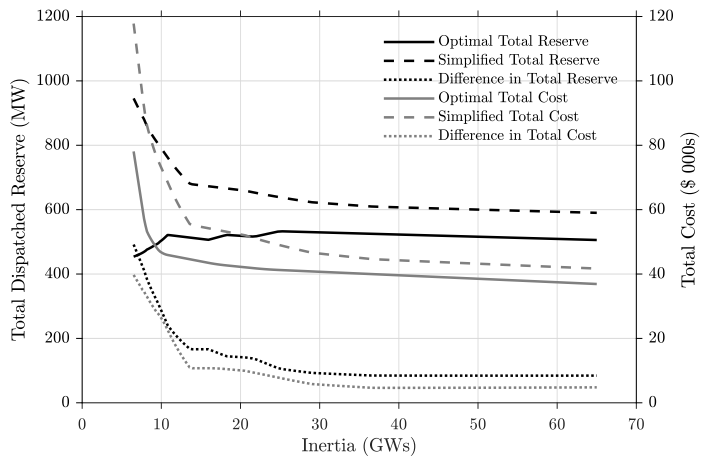

Fig. 4. Differences in total cost and total dispatched reserve for variations in inertia.

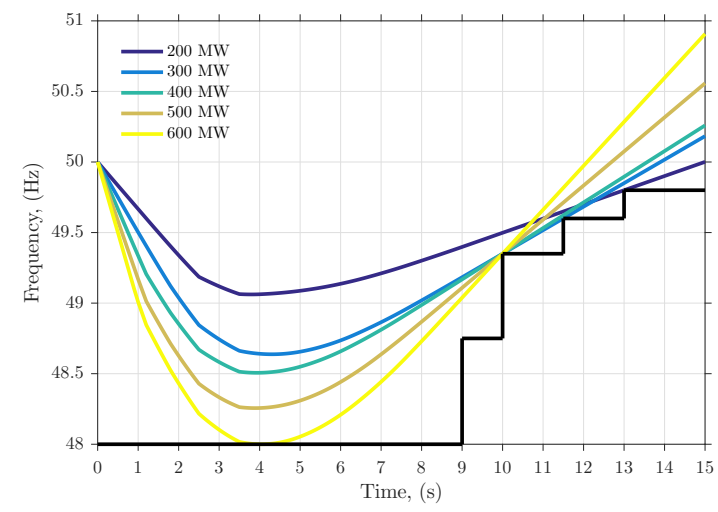

Fig. 5. Frequency transients for different risks.
TABLE VI

RESULTS FOR SECOND EXAMPLE FOR VARIATIONS IN INERTIA.

\begin{tabular}{|c|ccccc|}
\hline Offer & \multicolumn{5}{|c|}{ Inertia (MWs) } \\
& 6,500 & 11,559 & 20,555 & 36,552 & 65,000 \\
\hline \multicolumn{5}{|c|}{ IL Dispatch (MW) } \\
1 & 65.5 & 0 & 0 & 0 & 0 \\
2 & 16 & 0 & 0 & 0 & 0 \\
3 & 54 & 0 & 0 & 0 & 0 \\
4 & 152 & 112.4 & 73.2 & 42.4 & 23.1 \\
5 & 23 & 23 & 23 & 23 & 23 \\
6 & 15.0 & 89 & 89 & 89 & 89 \\
7 & 48 & 48 & 48 & 48 & 48 \\
\hline \multicolumn{5}{|c|}{ SR Dispatch (MW) } \\
1 & 24.8 & 18.4 & 19.9 & 21.4 & 21.4 \\
2 & 5.8 & 8.2 & 9.3 & 10.5 & 10.5 \\
3 & 6.3 & 14.3 & 16.6 & 18.9 & 18.9 \\
4 & 15 & 46.2 & 54.1 & 62.0 & 62.0 \\
5 & 17.1 & 81.9 & 97.1 & 112.3 & 112.3 \\
6 & 3.9 & 31.5 & 37.7 & 43.9 & 43.9 \\
7 & 1.7 & 18.5 & 22.1 & 25.8 & 25.8 \\
8 & 6 & 28 & 28 & 28 & 28 \\
\hline \multicolumn{5}{|c|}{ Total Dispatched Reserve (MW) } \\
& 454 & 519.3 & 518.7 & 525.0 & 505.7 \\
\hline \multicolumn{5}{|c|}{ Total Cost (\$) } \\
& 78,090 & 45,555 & 42,099 & 39,989 & 36,903 \\
\hline
\end{tabular}

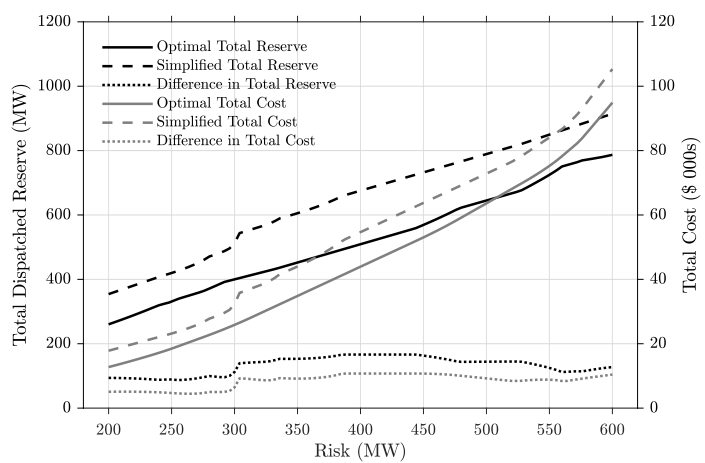

Fig. 6. Differences in total cost and total dispatched reserve for variations in risk.

The main point is that variations in inertia have a different impact in changing total cost compared to variations in risk. For changes in inertia above $10,000 \mathrm{MWs}$, the total cost remains relatively constant. For inertia less than 10,000 MWs, when the minimum frequency constraint becomes binding, faster more expensive reserve replaces slower reserve, and the total cost increases quickly. For changes in risk, there is a consistent change in total cost, this is reflected in the consistent change in total reserve required as risk increases. The implication this has on forming reserve markets is that risk should always be a variable in the optimization if it can vary, whereas for inertia it is less important. The inertial constant should only be an optimized variable if the inertia is likely to be below the knee point, at which the total cost is greater than $\$ 45,000$ for this example. The location of the knee point will depend on the risk, minimum frequency limit, and offers available, therefore actual data is required to localize this knee point in a real power system.

\section{COMPARING RESERVE Formulations}

To evaluate the benefit of considering the response time of reserve within the optimization, a 'simplified' formulation is 
TABLE VII

RESULTS FOR SECOND EXAMPLE FOR VARIATIONS IN RISK.

\begin{tabular}{|c|ccccc|}
\hline Offer & \multicolumn{5}{|c|}{ Risk (MW) } \\
& 200 & 300 & 400 & 500 & 600 \\
\hline \multicolumn{5}{|c|}{ IL Dispatch (MW) } \\
1 & 0 & 0 & 0 & 0 & 33.4 \\
2 & 0 & 0 & 0 & 0 & 16 \\
3 & 0 & 0 & 0 & 24.0 & 54 \\
4 & 0 & 0 & 102.2 & 152 & 152 \\
5 & 0 & 23 & 23 & 23 & 23 \\
6 & 89 & 89 & 89 & 89 & 89 \\
7 & 48 & 48 & 48 & 48 & 48 \\
\hline \multicolumn{5}{|c|}{ SR Dispatch (MW) } \\
1 & 0 & 6.9 & 18.4 & 42.4 & 69.4 \\
2 & 0 & 6.2 & 8.2 & 13.4 & 19.2 \\
3 & 0 & 13.0 & 14.3 & 19.8 & 25.9 \\
4 & 3.2 & 45.9 & 46.2 & 57 & 62 \\
5 & 43.7 & 85.5 & 81.9 & 94.5 & 108.7 \\
6 & 27.5 & 34.1 & 31.5 & 34.5 & 37.9 \\
7 & 20.9 & 20.4 & 18.5 & 19.4 & 20.4 \\
8 & 28 & 28 & 28 & 28 & 28 \\
\hline \multicolumn{5}{|c|}{ Total Dispatched Reserve (MW) } \\
& 260.3 & 400.0 & 509.1 & 644.9 & 786.9 \\
\hline \multicolumn{5}{|c|}{ Total Cost (\$) } \\
& 12,749 & 25,862 & 43,928 & 63,613 & 94,903 \\
\hline
\end{tabular}

created that only considers capacity of reserve. The optimal dispatch for the simplified formulation is obtained by finding all the least expensive reserve until the reserve requirement has been satisfied. In order to compare the formulations against the same frequency constraints, a routine is created for the simplified formulation, where the reserve requirement is adjusted so that the frequency limits just become binding. This is to approximate the optimization of reserve in New Zealand [8], and formulations like [9]. The results of this analysis are shown in Figs. 4 and 6 when comparing Example 2.

Although a benefit is seen for the optimal formulation over the simplified for all variations in inertia and risk, it is when the inertia is low that the greatest benefit is seen. For an inertial constant of 6,500 MWs in Example 2, the optimal formulation presents an improvement by reducing the total cost by $33.7 \%$ and the total dispatched reserve by $52.0 \%$. The average reduction in total dispatched reserve across all cases considered is $22.7 \%$, which is a more accurate reflection of the overall benefit of the optimal formulation.

\section{Pricing Methodology}

A pricing methodology is developed from the principle of marginal pricing, e.g. [9], [10]. In the reserve optimization, the response characteristics of reserve providers over time are captured. Therefore, the marginal reserve price is not a single value, but a function of time when a unit of reserve responds. The marginal reserve price is analyzed by considering the KKT condition on the gradient of the boundary functions, [11), at the point of the global minimum, $\mathbf{v}^{*}=\left[H^{*}, R^{*}, p_{1}^{*}, \ldots, p_{N_{p}}^{*}, u_{1}^{*}, \ldots, u_{N_{u}}^{*}\right]^{T}$.

The variables $\nu$ and $\lambda_{j}$ are the KKT multipliers for the reserve requirement constraint, $R(\mathbf{v}) \leq 0$, and the frequency limits, $F_{j}(\mathbf{v}) \leq 0$, respectively. The minimum frequency constraint requires special consideration, due to it being a non-smooth function when the frequency transient is flat at its minimum, e.g. Fig. 5 with 6,500 MWs of inertia. Therefore additional gradients, $\nabla F_{\min , j}\left(\mathbf{v}^{*}\right)$, are possible at the point $\mathbf{v}^{*}$, where $t_{\min }$ can jump to $t_{m i n, j}$, that is:

$$
\Omega^{*}=\left\{t \mid \exists i t=t_{i, p} \text { or } t=t_{i, u}, f^{*}(t)=f_{\text {lim }}(t)\right\}
$$

The KKT multipliers $\boldsymbol{\mu}^{\min }$ and $\boldsymbol{\mu}^{\max }$ are for the minimum and maximum limits on inertia, risk, and the amount of reserve dispatched. All KKT multipliers can be obtained from the optimal dual variables to the solved region from which the global minimum occurs. Lastly the cost vector is $\mathbf{c}=\left[0,0, c_{1, p}, \ldots, c_{N_{p}, p}, c_{1, u}, \ldots, c_{N_{u}, u}\right]^{T}$.

The gradient for frequency constraints share a similar structure and is generalized by $\mathbf{G}\left(f_{k, \lambda}, t_{k}, \boldsymbol{\tau}\right)$ :

$$
\begin{gathered}
\mathbf{G}\left(f_{\text {lim }}, t_{\text {min }}^{*}, \boldsymbol{\tau}^{*}\right)=\nabla F_{\min }\left(\mathbf{v}^{*}\right) \\
\mathbf{G}\left(f_{l i m, j}, t_{m i n, j}^{*}, \boldsymbol{\tau}^{*}\right)=\nabla F_{\text {min }, j}\left(\mathbf{v}^{*}\right) \\
\mathbf{G}\left(f_{j}, t_{j, l}, \boldsymbol{\tau}^{*}\right)=\nabla F_{j}\left(\mathbf{v}^{*}\right)
\end{gathered}
$$

where

$G_{i}\left(f_{k, \lambda}, t_{k}, \boldsymbol{\tau}\right)= \begin{cases}2 f_{k, \lambda} & i=1, \text { inertial component } \\ t_{k} & i=2, \text { risk component } \\ -\left(t_{k}-\tau_{i}\right) & \text { offer } i \text { where } \tau_{i} \leq t_{k} \\ 0 & \text { offer } i \text { where } \tau_{i}>t_{k}\end{cases}$

and $\tau$ is a vector of times when a reserve offer responds:

$$
\tau_{i}= \begin{cases}t_{i, p} & \text { where } i \text { is an IL offer } \\ t_{i, u}+u_{i} / g_{i} & \text { where } i \text { is a SR offer }\end{cases}
$$

The marginal reserve price function is created by considering the relationship between reserve price in (11) and the time a unit of reserve is offered $\tau$ for the reserve components of (11), while $\mu_{i}^{\min }=0$ and $\mu_{i}^{\max }=0$. The marginal reserve price function is:

$$
c(\tau)=\nu+\sum_{\substack{k \\ \tau \leq t_{k}}} \lambda_{k}\left(t_{k}-\tau\right)
$$

where $t_{k} \in\left\{t_{\text {min }}^{*}, t_{\text {min }, 1}^{*}, \ldots, t_{1, l}, t_{2, l}, \ldots\right\} \quad$ and has its corresponding KKT multiplier $\lambda_{k} \in$ $\left\{\lambda_{\min }, \lambda_{\min , 1}, \ldots, \lambda_{1, l}, \lambda_{2, l}, \ldots\right\}$. The marginal reserve price function is shown for Example 2, for variations in inertia in Fig. 7 and for variations in risk in Fig. 8 All the reserve that is dispatched is offered at a time and price below the marginal reserve price function. Therefore if a reserve provider desires to increase its dispatched quantity, then it has to provide additional units of reserve with a time and price below $c(\tau)$.

Out of Example 2, the most complicated marginal reserve price function is for $6500 \mathrm{MWs}$, as shown in Fig. 7 The formulation of its curve is demonstrated by the values in Table VIII.

The marginal cost of risk is $c_{R}=c(0)$, which can be found as y-intercepts in Figs. 7 and 8 The marginal value of inertia is:

$$
c_{H}=-2 \sum_{k} \lambda_{k} f_{k, \lambda}
$$




$$
\mathbf{c}+\nu \nabla R\left(\mathbf{v}^{*}\right)+\lambda_{\min } \nabla F_{\min }\left(\mathbf{v}^{*}\right)+\sum_{t_{m i n, j}^{*} \in \Omega^{*}} \lambda_{\min , j} \nabla F_{\min , j}\left(\mathbf{v}^{*}\right)+\sum_{j=1}^{N_{c}} \lambda_{j, l} \nabla F_{j}\left(\mathbf{v}^{*}\right)-\boldsymbol{\mu}^{\min }+\boldsymbol{\mu}^{\max }=0
$$

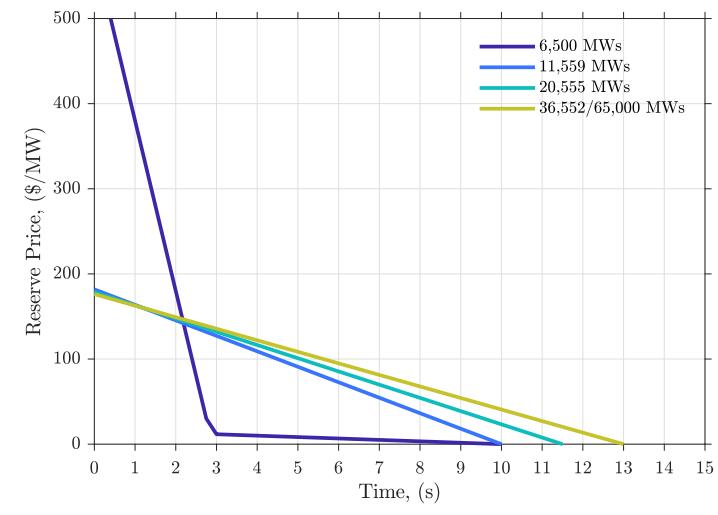

Fig. 7. Marginal reserve price function for Example 2 with different inertial constants. For inertia values of 36552 and 65000 MWs the marginal price function shares the same profile.

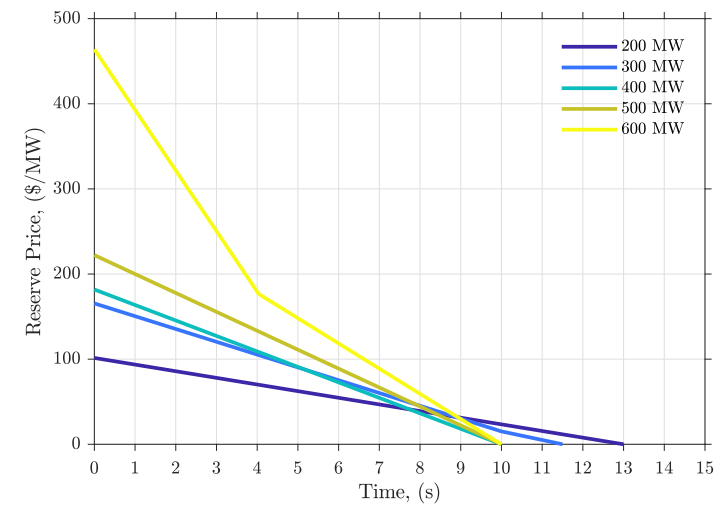

Fig. 8. Marginal reserve price function for Example 2 with different risk, i.e. size of credible contingency.

where $f_{k, \lambda} \in\left\{f_{\text {lim }}, f_{\text {lim }, 1}, \ldots, f_{1}, f_{2}, \ldots\right\}$. The value $c_{H}$ can form part of the price for inertia, if a inertia market is desired in the co-optimized problem. The values for $c_{H}$ for the different cases of Example 2 are shown in Table IX

An IL offer is remunerated with a value equal to $p_{i}^{*} c\left(t_{i, p}\right)$. For a SR offer, remuneration requires considering the time each unit of reserve responds:

$$
\int_{t_{i, u}}^{t_{i, u}+\frac{u_{i}^{*}}{g_{i}}} g_{i} c(\tau) d \tau
$$

In Table $\mathrm{X}$ the remuneration of reserve providers is shown for Example 2, with an inertial constant of 15,000 MWs and a risk of $400 \mathrm{MW}$. It is seen that each reserve provider is paid at a price related to how quickly it responds. An incentive is created for participants to improve response speed in order to receive a higher price.
TABLE VIII

Parameters for Marginal Reserve PRICE Function for the 6500 MWS CASE OF EXAMPLE 2.

\begin{tabular}{|c|ccc|}
\hline $\begin{array}{c}\text { Time Variable of Binding } \\
\text { Frequency Constraint }\end{array}$ & $t_{k}(\mathrm{~s})$ & $\lambda_{k}(\$ / \mathrm{MWs})$ & $f_{k, \lambda}$ \\
\hline$t_{\min }$ & 2.75 & 126.67 & -0.04 \\
$t_{\min , 1}$ & 3.00 & 71.67 & -0.04 \\
$t_{2, l}$ & 10.00 & 1.67 & -0.013 \\
\hline
\end{tabular}

TABLE IX

INERTIA PRICES FOR THE SECOND EXAMPLE.

\begin{tabular}{|c|ccccc|}
\hline & \multicolumn{5}{|c|}{ Variations in Inertia } \\
$H$ (MWs) & 6,500 & 11,559 & 20,555 & 36,552 & 65,000 \\
$c_{H}(\$ / \mathrm{MWs})$ & 15.91 & 0.47 & 0.25 & 0.11 & 0.11 \\
\hline & \multicolumn{5}{|c|}{ Variations in Risk } \\
$R$ (MW) & 200 & 300 & 400 & 500 & 600 \\
$c_{H}(\$ / \mathrm{MWs})$ & 0.06 & 0.29 & 0.47 & 0.58 & 4.10 \\
\hline
\end{tabular}

\section{DISCUSSION}

The representation of reserve as instantaneous and ramped responses requires further explanation. Standard linear control methods for generators includes a droop setting and the dynamics can be modeled by one time constant [11], where the power system frequency is the input for the controller. This control method ensures the stability of a power system, but makes it difficult to optimize the availability of reserves. The proposed optimization methodology models reserve response with a feed-forward approach. To model a conventional generator with a feedback controller, it is necessary to test the generator's response with a standard frequency transient, as is commonly used for establishing the capabilities of reserve providers [12]. [13] provides a discussion on the relationship between a standard frequency transient and ensuring the minimum frequency constraint. It also should be mentioned that a generator controller can be suspended in fast declining frequency events, instead the reserve provider can be ordered to maximum power output as quickly as possible. Once the frequency has recovered the original controller can be reengaged to ensure long-term stability. This control structure is already in place in some New Zealand generators [14]. Therefore, a wide range of frequency transients could result in the same reserve response, for which this optimization methodology is well suited.

After testing the reserve provider it may be found that the response does not fit an instantaneous or ramped response very accurately. In these situations, it is possible to apply multiple ramped offers to approximate the reserve response, as seen in Fig. 9 approximating the curve with multiple straight line segments. A condition is required: later offers have to have higher prices than earlier offers, to ensure reserve is dispatched in sequence. Similar methods can also approximate the response of load-damping, as it can be modeled by multiple SR offers with zero price. 
TABLE $X$

COMPARING THE REMUNERATION OF RESERVE FOR OPTIMAL DISPATCH AND THE SIMPLIFIED DISPATCH

\begin{tabular}{|c|c|c|c|c|c|}
\hline \multirow[b]{2}{*}{ Offer } & \multicolumn{3}{|c|}{ Optimal Dispatch } & \multicolumn{2}{|c|}{$\begin{array}{c}\text { Simplified Dispatch } \\
\text { Reserve Price } \$ 160 / \mathrm{MW}\end{array}$} \\
\hline & $\begin{array}{l}\text { Dispatch } \\
\text { (MW) }\end{array}$ & $\begin{array}{c}\text { Average Price } \\
\text { (\$/MW) }\end{array}$ & $\begin{array}{c}\text { Charge } \\
(\$)\end{array}$ & $\begin{array}{l}\text { Reserve } \\
\text { Dispatch } \\
\text { (MW) }\end{array}$ & $\begin{array}{l}\text { Charge } \\
\text { Ch) }\end{array}$ \\
\hline IL & & & & & \\
\hline 1 & 0 & 165.5 & 0 & 0 & 0 \\
\hline 2 & 0 & 163.6 & 0 & 0 & 0 \\
\hline 3 & 0 & 163.6 & 0 & 0 & 0 \\
\hline 4 & 102.2 & 160 & 16,355 & 16.5 & 2,636 \\
\hline 5 & 23 & 149.1 & 3,429 & 23 & 3,680 \\
\hline 6 & 89 & 136.4 & 12,136 & 89 & 14,240 \\
\hline 7 & 48 & 118.2 & 5,673 & 48 & 7,680 \\
\hline \multicolumn{6}{|l|}{ SR } \\
\hline 1 & 18.4 & 160.5 & 2,952 & 71 & 11,360 \\
\hline 2 & 8.2 & 148.6 & 1,219 & 26 & 4,160 \\
\hline 3 & 14.3 & 135.9 & 1,937 & 67 & 10,720 \\
\hline 4 & 46.2 & 125 & 5,775 & 62 & 9,920 \\
\hline 5 & 81.9 & 111.4 & 9,121 & 165 & 26,400 \\
\hline 6 & 31.5 & 97.7 & 3,078 & 47 & 7,520 \\
\hline 7 & 18.5 & 85.9 & 1,585 & 33 & 5,280 \\
\hline \multirow[t]{3}{*}{8} & 28 & 84.8 & 2,376 & 28 & 4,480 \\
\hline & \multicolumn{5}{|c|}{ Totals and Average Price } \\
\hline & 509.1 & 128.9 & 65,636 & 675.5 & 108,076 \\
\hline
\end{tabular}

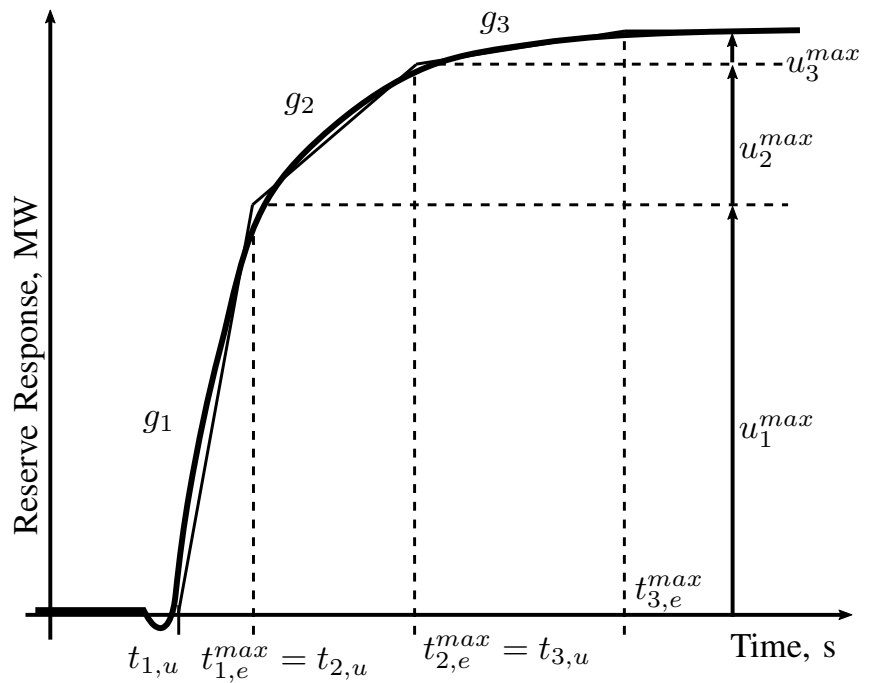

Fig. 9. Approximating the reserve response of hydro generator to a step change in frequency with three SR offers.

Some reserve providers cannot sustain a positive reserve output indefinitely. It is not possible to model their response with the proposed positive instantaneous and ramped offers. For example, a wind turbine operating at its maximum output can provide extra power output by slowing down its rotor, but this cannot be sustained indefinitely as the maximum power point has to be found again. HydroQuebec requires this response from wind turbines in their system [15], and Ireland has created Fast Frequency Response as a reserve category for these providers [1], so it is important not to deny access to these providers. The simplest way to include wind reserve is to allow for negative instantaneous reserve offers, an instantaneous reduction in power output, into the formulation. This will make it possible to optimize reserve from wind turbines through combining multiple positive and negative reserve offers. Since it is known that the instantaneous offers only present linear terms to the constraints, the space confined by the frequency limits is convex, and the feasible solution space can be divided into finitely many regions which are also convex. There can now be multiple minimum frequency constraints. However, it is unknown whether the union of all these regions gives a convex feasible solution space. The introduction of negatively ramped offers is expected to be significantly more difficult.

In Section II the importance of the frequency limits in returning the frequency to nominal is shown. However, it is not clear when these frequency limit step changes should occur and to which level. Limits on duration for how long the frequency can be below a certain level exist for generators, but before these frequencies are reached the frequency settings for emergency load shedding occur, and before this the minimum frequency for credible contingencies. This is one clear limit for the proposed reserve formulation. In New Zealand, [16] requires the frequency to return to $50 \pm 0.75$ $\mathrm{Hz}$ one minute after a contingency. This could provide a second limit, except this leaves one minute with no other restriction on the frequency transient. Clearly analysis of the most efficient frequency limits is required, which considers the cost of procuring additional reserve against the benefit of additional security. The proposed reserve optimization can be repurposed so that instead of inertia being a decision variable, the frequency levels, $f_{j}$, become variables. The feasible solution space remains convex and the same solving methodology can be applied. This analysis is similar to adding dynamic constraints into an optimization by [17] to find the optimal frequency settings for emergency load shedding blocks.

\section{CONCLUSION}

This paper has shown it is theoretically possible to construct a real-time co-optimized energy and reserve market that can optimize inertia, risk, and various types of reserve with different response speeds. A fully co-optimized example has not been shown, but it can be inferred from the convexity of reserve optimization problem and the amount of time required to solve these problems that it is possible to construct a practical mixed integer solver for a real-time market. The benefit of developing this methodology is to improve the way decisions are made between changing inertia requirements for power systems, the management of risk, and the construction of new reserve providers. It is seen that the proposed methodology can reduce the total reserve requirement over methodologies that only consider the capacity of reserve in optimization. A pricing methodology is developed to price reserve by the time each unit is offered. This provides an incentive for participants to improve response speed.

Further analysis is required to determine the timing and level of frequency limits as no sufficient standard exists in this range. It is shown that it is possible to find the optimal solution, if one frequency constraint is binding, by the formation of a merit-order curve. This provides a simple means of understanding the optimal solution, which aids market participation. In the second example, the impact of variations in inertia and risk on total cost of the optimal solution are 
analyzed. The necessity of having the inertial constant as a decision variable is markedly less than that of risk, i.e. the largest credible contingency. Further analysis on real power systems is required to validate this observation, and determine when the inertial constant should be a decision variable in the optimization rather than a parameter.

\section{REFERENCES}

[1] SEM, "DS3 System Services Technical Definitions, Decision Paper," Single Electricity Market, Ireland, Tech. Rep. SEM-13-098, Dec, 2013.

[2] National Grid, "Enhanced Frequency Response, Invitation to tender for pre-qualified parties," United Kingdom, Tech. Rep., Jul, 2016.

[3] AEMO, "Fast Frequency Response in the NEM," Australian Energy Market Operator, Australia, Tech. Rep., 2017.

[4] J. Schipper, A. Wood, C. Edwards, "Optimizing Instantaneous and Ramping Reserves with Different Response Speeds for ContingenciesPart I: Methodology", IEEE Trans. Power Sys., to be published.

[5] Y. Rebours, A Comprehensive Assessment of Markets for Frequency and Voltage Control Ancillary Services, PhD Thesis, School of Electrical and Electronic Engineering, University of Manchester, United Kingdom, 2008.

[6] System Operator, "SPD Schedule Inputs," Transpower, Wellington, New Zealand, Tech. Rep. GL-OC-209, May, 2018.

[7] J. Schipper, "Optimising Power System Reserve for Contingencies while considering Response Times," PhD Thesis, Department of Electrical and Computer Engineering, University of Canterbury, Christchurch, New Zealand, 2019.

[8] System Operator, "Reserve Management Tool Functional Specification", Transpower, Wellington, New Zealand, 2016.

[9] E. Ela, V. Gevorgian, A. Tuohy, B. Kirby, M. Milligan, and M. O'Malley, "Market Designs for the Primary Frequency Response Ancillary ServicePart I: Motivation and Design," IEEE Trans. Power Sys., vol. 29, no. 1, pp. 421-431, 2014.

[10] System Operator, UG-SD-025 SPD_Model_Formulation_v10, Transpower, Wellington, New Zealand, 2016.

[11] P. Kundur, Power System Stability and Control, New York, NY, USA: McGraw-Hill, 1994.

[12] Transpower, "Companion Guide for Testing of Assets," Wellington, New Zealand, Tech. Rep. GL-EA-010, August, 2016.

[13] L. E. Sokoler, P. Vinter, R. Baerentsen, K. Edlund, and J. B. Jorgensen, "Contingency-constrained unit commitment in meshed isolated power systems," IEEE Trans. Power Sys., vol. 31, no. 5, pp. 3516-3526, 2016.

[14] N. Vong, M. Phethean, and S. Nutt, "Managing system frequency dynamics in small power system by hydro generating unit operated in Tail Water Depress (TWD) mode with use of Feed Forward Controls," in Proc. IEEE PES General Meeting, July, 2010.

[15] M. Asmine, C. Langlois, and N. Aubut, "Inertial Response from wind power plants during a frequency disturbance on the HydroQuebec system - event analysis and validation," IET Renew. Power Gener, vol. 12, no. 5, pp. 515-522, 2018.

[16] Electricity Authority, "Policy Statement, Electricity Industry Participation Code", Wellington, New Zealand, 2018.

[17] S. S. Banijamali, T. Amree, "Semi-Adaptive Setting of Under Frequency Load Shedding Relays Considering Credible Generation Outage Scenarios," IEEE Trans. Power Del., vol. 34, no. 3, pp. 1098-1108, 2019. ,

Josh Schipper received the B.E. and Ph.D. degrees in electrical and electronic engineering from the University of Canterbury, Christchurch, New Zealand, in 2014 and 2019 , respectively.

Alan Wood (M'96) received the B.E. and Ph.D. degrees in electrical and electronic engineering from the University of Canterbury, Christchurch, New Zealand, in 1981 and 1993, respectively. Currently, he is an Associate Professor at the University of Canterbury.

Conrad Edwards received the MA (Hons) in Operational Research from Cambridge University, United Kingdom. He has been working in Transpower New Zealand Limited since 1998. 